\title{
Defining matrix Gla protein expression in the Dunkin-Hartley guinea pig model of spontaneous osteoarthritis
}

\author{
Xun Ma ${ }^{1 \dagger}$, Zhan Zhang ${ }^{2 \dagger}$, Xinyuan Kang ${ }^{2 \dagger}$, Chunbo Deng ${ }^{2,3}$, Yingwei Sun ${ }^{4,5}$, Yanjun Li ${ }^{6}$, Desheng Huang ${ }^{6}$ and
} Xueyong Liu ${ }^{1 *}(\mathbb{Z}$

\begin{abstract}
Background: Matrix Gla ( $\gamma$-carboxyglutamate) protein (MGP) is considered a strong inhibitor of ectopic calcification, and it has been associated with OA severity, although not conclusively. We utilized male Dunkin-Hartley (DH) guinea pigs to investigate the expression of MGP throughout aging and disease pathogenesis in a spontaneous model.

Method: Twenty-five male DH guinea pigs were obtained and nurtured to several timepoints, and then randomly and equally divided by age into five subgroups (1-, 3-, 6-, 9-, and 12-months, with the 1-month group as the reference group). DH guinea pigs in each group were euthanized at the designated month-age and the left or right medial tibial plateaus cartilages were randomly excised. OA severity was described by modified Mankin Score (MMS) at microscopy (Safranin O/Fast Green stain). Proteomic evaluation using isobaric tags for relative and absolute quantification (iTRAQ) was performed to validate the age-related changes in the MGP profiles, and immunohistochemistry (IHC) methods were applied for semi-quantitative determination of MGP expression in articular cartilage.
\end{abstract}

Results: The histopathologic findings validated the increasing severity of cartilage degeneration with age in the $\mathrm{DH}$ guinea pigs. The MMS showed significant, stepwise (every adjacent comparison $P<0.05$ ) disease progression with month-age. The iTRAQ indicated that MGP levels increased significantly with advancing age $(P<0.05)$, as supported by the $\mathrm{IHC}$ result $(\mathrm{P}<0.05)$.

Conclusion: Increased expression of MGP in male DH guinea pigs was present throughout aging and disease progression and may be link to increased OA severity. Further studies are needed to investigate and confirm the association between MGP levels and OA severity.

Keywords: Matrix Gla protein, Osteoarthritis, Guinea pig, Proteomics

*Correspondence: liusjh@sj-hospital.org

${ }^{+}$Xun Ma, Zhan Zhang and Xinyuan Kang contributed equally to this work.

${ }^{1}$ Department of Rehabilitation, Shengjing Hospital of China

Medical University, No.16, Puhe Street, Shenyang North New Area, Shenyang 110134, Liaoning Province, China

Full list of author information is available at the end of the article

\section{Introduction}

Osteoarthritis (OA) is the most common degenerative joint disease of the middle-aged and elderly [1]. OA may lead to reduced quality of life, and it is a major cause of work incapability and disability. OA is an important medical challenge across the globe [2], but the etiology, risk factors, and pathophysiology remain unclear. At present, early detection and treatment of OA are important. Identifying new therapeutic targets and biomarkers of $\mathrm{OA}$ is a original author(s) and the source, provide a link to the Creative Commons licence, and indicate if changes were made. The images or other third party material in this article are included in the article's Creative Commons licence, unless indicated otherwise in a credit line to the material. If material is not included in the article's Creative Commons licence and your intended use is not permitted by statutory regulation or exceeds the permitted use, you will need to obtain permission directly from the copyright holder. To view a copy of this licence, visit http://creativecommons.org/licenses/by/4.0/. The Creative Commons Public Domain Dedication waiver (http://creativeco mmons.org/publicdomain/zero/1.0/) applies to the data made available in this article, unless otherwise stated in a credit line to the data. 
research focus, but biomarkers of OA severity are rarely reported.

Matrix Gla ( $\gamma$-carboxy glutamate) protein (MGP) is a vitamin $\mathrm{K}$-dependent protein that was first isolated from bovine bone [3]. Uncarboxylated (inactive) MGP is converted into carboxylated (active) MGP by vitamin $\mathrm{K}$, and by this way it is possible to detect the vitamin $\mathrm{K}$ status in vivo [4]. MGP may function as an inhibitor of ectopic calcification in cardiovascular tissues and cartilage, but the detailed mechanism remains unclear. MGP has been shown to inhibit BMP-2-induced calcification and spontaneous vascular calcification [5], and MGP mutation may lead to Keutel syndrome, a genetic disorder that is mainly characterized by multiple pathological changes including cartilage calcification, brachydactyly, and pulmonary artery stenosis [6]. Meanwhile, MGP was reported as an anti-inflammation cytokine, may have the effects of alleviating inflammatory reaction in patients with acute pancreatitis and arthritis [7, 8]. rs1800802 located in an MGP polymorphic site has been reported in association with an increased OA risk in a Han Chinese population $[9,10]$. However, although a potential association between MGP and OA has also been suggested, there is still no consensus or detailed mechanism regarding the presence and effect of MGP in OA severity and natural history.

The Dunkin-Hartley (DH) guinea pig is considered a useful and common animal model of spontaneous $\mathrm{OA}$, with several features that are similar to human OA pathogenesis and progression $[11,12]$. Based on previous studies [13, 14], MGP is hypothesized to be associated with OA pathogenesis and/or prognosis. Thus, this study was conducted to investigate MGP levels in a naturallyoccurring model at key points throughout aging.

\section{Materials and methods}

\section{Animal experiments}

All animal experiments were performed with approval of the Animal Ethics Committee of Shengjing Hospital of China Medical University (No. 2016PS284K), which complies with the Guide for the Care and Use of Laboratory Animals, 8th edition, published by the United States National Institutes of Health (NIH Publication, 2011) [15] and Animal Research: Reporting of In Vivo Experiments (ARRIVE) Guidelines 2.0 [16]. The ARRIVE checklist was available in Supplementary Materials.

A total of 25 male DH guinea pigs with healthy appearance, appetite and activity were obtained from Beijing Huafukang Bioscience Co. Inc. Each month (1-, 3-, 6-, 9-, and 12-months), 5 guinea pigs were randomly selected as the marked month-age group by random number table, with the 1-month-old guinea pigs as the reference group, other month-olds as observation groups. The animals were nurtured in the Animal Department of Shengjing Hospital Affiliated to China Medical University. Every random two guinea pigs in each cage were kept and drank freely. The indoor temperature was controlled at $20^{\circ} \mathrm{C}-25^{\circ} \mathrm{C}$ and the humidity was kept at $40-50 \%$. The $\mathrm{DH}$ guinea pigs were conventional feeding with standard guinea pig chow (purchased from Shenyang Maohua Biotechnology Co., Ltd), and for the need of vitamin C, they were fed with fresh vitamin C-rich cabbage every day for the first week and 2-4 times for the rest weeks.

All the experimental procedures were performed in the Experimental Center of Shengjing Hospital of China Medical University. During the entire study period, the investigators in charge of animal raising were blind for all the subsequent operations involving animals, and the investigators performed the experiment were not aware of the grouping. When the groups reached the target age, the animals were weighed. The guinea pigs were then placed under general anesthesia by intraperitoneal injection of $3 \%$ pentobarbital sodium, followed by intact knee specimens obtained, and then guinea pigs were then euthanized by intracardiac air injection embolization. For every guinea pig in a certain group, a randomly determined left or right-side knee specimen was selected for proteomics analysis, and the other side knee were used for the immunohistochemistry (IHC) examination in animal experiments; and, cartilages of medial tibial plateau are used in both of IHC and proteomics procedures. The gross specimens were photographed with a Canon 5D Mark3 camera.

\section{Proteomics analysis}

After gross knee specimens were collected from all groups, the knee specimens were immediately put into a $4^{\circ} \mathrm{C}$ incubator and randomly taken photographs, temporary preserved in liquid nitrogen and quickly transferred to a $-80^{\circ} \mathrm{C}$ refrigerator for long-term preservation. Cartilages of medial tibial plateau obtained from a random lower limb of every guinea pig were sampled under liquid nitrogen environment, and interested cartilages of each group were mixed together for the proteomic analyses. The total protein of the interested cartilages of each group was extracted by a protein extraction kit. Isobaric tags for relative and absolute quantification (iTRAQ) tests were performed in triplicate in each group, and stepwise comparisons of differentially expressed proteins (DEPs) were made between sequential groups to identify proteins with change of 1.2 -fold or greater $(P<0.05)$.

\section{Histopathologic analysis}

Total knee specimens without cartilage extraction were stored in $4 \%$ paraformaldehyde for one week, then the specimen was washed with running water for $12 \mathrm{~h}$, and 
then decalcified in EDTA decalcification solution. The decalcification was at $25^{\circ} \mathrm{C}$ and the durations depended on the size of the specimen. The decalcification time of knee joint specimens of 1-month-old guinea pigs was about 4 weeks, and that of 12-month-old guinea pigs was 12 weeks. The rest month-olds were between 4 and 12 weeks. After complete decalcification, the gross knee joint was sectioned from the medial collateral ligament along the coronal plane. The wax-blocking procedure is as follows: 1. Wash with running water for $8 \mathrm{~h}, 75 \%$ alcohol overnight, $85 \%$ alcohol for $3 \mathrm{~h}, 95 \%$ alcohol (I) for $3 \mathrm{~h}, 95 \%$ alcohol (II) for $2 \mathrm{~h}, 100 \%$ alcohol (I) for $2 \mathrm{~h}$, $100 \%$ alcohol (II) for $2 \mathrm{~h}$, xylene (I) for $30 \mathrm{~min}$, xylene (II) for $30 \mathrm{~min}$, paraffin (I) for $60 \mathrm{~min}$, paraffin (II) for $90 \mathrm{~min}$. Then the wax block was made by tissue embedding machine. The embedded wax blocks were sliced with a paraffin section machine with a thickness of $3 \mu \mathrm{m}$. Pick up the chips, bake them in a $60^{\circ} \mathrm{C}$ toaster, and bake them in a $60^{\circ} \mathrm{C}$ oven overnight.

Then Safranine O-fast green staining was performed. Medial tibial plateau area was chosen for histopathologic analysis due to reported as the earliest-phase and most common degeneration region in knee OA. The severity of OA was graded according to a modified Mankin score (MMS) [17, 18]. The MMS comprised four sections: Structure, Cellularity, Matrix staining, Tidemark integrity. Correlation analysis is used for the relationship between MMS and IOD/area. All sections were estimated blindly by an observer, triplicate values were performed for MMS.

\section{Immunohistochemistry (IHC)}

Coronal sections of the knee were prepared and used for the IHC analysis, and medial tibial plateau area was used due to the statements mentioned above. For the antigen retrieval process, the paraffin section was placed in sodium citrate buffer ( $\mathrm{pH} 7.2$ ), heated with high fire level in microwave oven for $7 \mathrm{~min}$, then cooled to room temperature for subsequent operation. After determining antigen and blocking endogenous peroxidase activity, obtained sections were incubated overnight with primary antibodies (anti-MGP, Sigma, SAB2101477, 1:50) at $4{ }^{\circ} \mathrm{C}$. A secondary antibody with peroxidase-linkage was then added for $30 \mathrm{~min}$ at room temperature. 3,3'-diaminobenzidine tetrahydrochloride (DAB) was applied to detect staining. The sections were then immediately dehydrated with progressively ascending concentrations of ethanol solutions, cleared with xylene, and mounted with a coverslip of DePeX medium. IPP (version 6.0) image analysis software was used for semi-quantitative IHC analysis of MGP expression by using the average optical density (OD) (IOD/sum) value to indicate the depth of staining of MGP (+) particles in the chondrocytes, with deeper browns indicating higher levels of MGP expression. Negative control was conducted with only secondary antibody utilized, and 1-month group was used as reference group.

\section{Statistical analysis}

All results are expressed as means \pm standard deviation (SD). SPSS version 23.0 (IBM, Rochester, MN, USA) was used for all statistical analyses. Differences between means were analyzed by one-way analysis of variance (ANOVA) when time was the independent variable and applied data were variance homogeneous and conformed normal distribution. Correlation analysis was used for the relationship between MMS and IOD/area.

Inter- and intra-observer agreement were measured with the intraclass correlation coefficient (ICC) [19], single measurements, and absolute agreement. Generally, ICC 0.70 is set as a minimum standard for test-retest reliability, with ICC $<0.40$ indicating weak correlation, 0.41 to 0.60 moderate correlation, 0.61 to 0.80 substantial correlation, and $>0.81$ describing near-perfect agreement. Statistical significance was set at $P<0.05$ for all analyses.

\section{Results}

\section{Characteristics of the guinea pigs}

A total of $25 \mathrm{DH}$ guinea pigs were included in this study. From baseline to specimen collection, all of the guinea pigs were in healthy appetite and activity, and no unplanned death occurred. The weight changes of the guinea pigs from 1-month to 12 -months are shown in Fig. 2 and Table 2.

\section{Histopathologic analysis}

Macroscopically, the articular surface was smooth and intact in the reference group, and a small amount of colorless and transparent synovial fluid could be seen in the articular cavity. As the guinea pigs aged, the articular fluid gradually became turbid and yellow and the articular surface showed gradual degeneration with cartilage slightly depressed. In the oldest group (12-months), the articular cartilage was rough and dull, with a thinner cartilage matrix and more cartilage depression, and osteophyte formation was visible at the edges of the joint. (Fig. 1 and Table 1), high quality figures with size marker were provided in Supplementary Materials.

At microscopical sights, no pathological manifestations of OA were observed in the reference group. For the cartilage structures of medial tibial plateau cartilage, tissue sections from the reference group showed non-degenerated cartilage structure, chondrocytes, and staining of the matrix, and no manifestations of degenerative disease were observed. At 3-months, the articular surface showed only tiny cracks with occasional cell aggregation and 


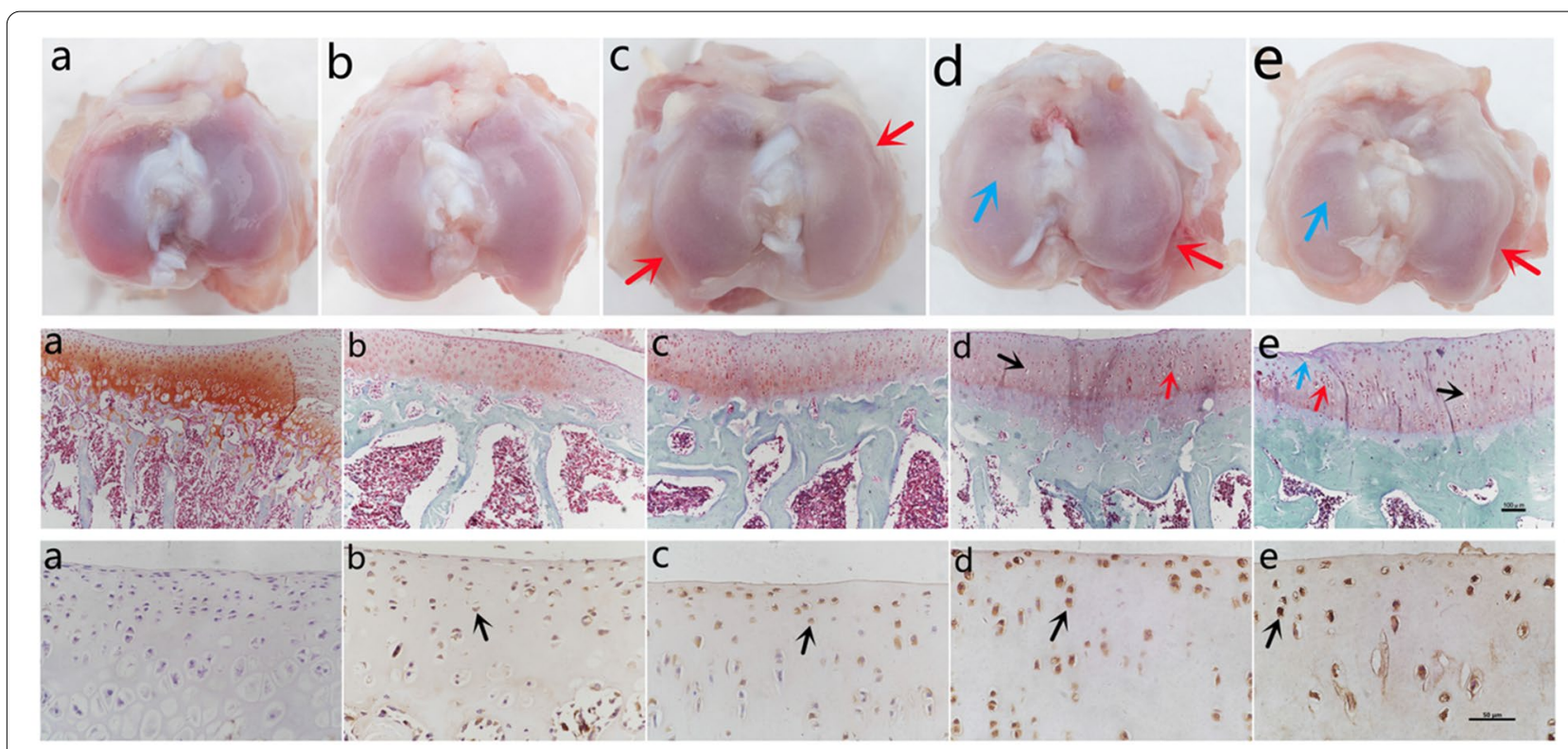

Fig. 1 Histopathological progression of spontaneous osteoarthritis with aging in the DH guinea pig model. Row 1 (a) to (e) shows the gross histopathological changes in the knee joint from 1-month to 12-months. Red arrow designated rough surface of knee cartilage, and orange arrows indicated degenerative area. Row 2 (a) to (e) shows the microscopic changes with aging in safranine O-fast green-stained medial tibial plateau cartilages (respectively 1-, 3-, 6-, 9- and 12-month-old). Black arrows designated loss of chondrocytes in lacunas, purple arrows indicated chondrocyte cluster, and green arrows meant multiple cracks of cartilage surface. Row 3 (a) to (e) shows the progressive increase in MGP levels detected by immunohistochemistry, from 1-month to 12-months in medial tibial plateau cartilages. Yellow arrows designate positive MGP-expressed chondrocytes

cloning, and no cell morphology or matrix abnormalities were seen. At 6-months to 9-months, progressive damage was indicated by roughened cartilage surface and irregular cartilage layer structure, the number of chondrocytes was significantly decreased, and necrosis and cell clusters and clones were gradually increased. By 12 months of age, the cartilage structure of knee was severely damaged and the number of chondrocytes was significantly decreased. In addition, the number of empty cartilage vesicles was increased, and the staining of the matrix was generally lost. (Fig. 1 and Table 1). These histopathological manifestations were in accordance with previous studies [20, 21]. The histopathological changes were analyzed semiquantitatively by MMS (Fig. 2 and Table 2), these were continuous data obtained after scoring conform to the normal distribution and conformed normal distribution. The score increased continuously from 1-month (reference group) to 12-months, and the increase was significant between every adjacent group $(P<0.05)$.

\section{Proteomics}

Proteomics analysis was performed by three-time repeat iTRAQ to detect DEPs over time. Respectively 1221, 1413, and 1400 proteins were screened in the three iTRAQ runs, and the results confirmed a general increase in MGP expression over time, significantly 1.2-fold changes of MGP levels were detected in two of three times (Tables 2 and 3).

\section{Immunohistochemistry}

The cytoplasm of MGP-positive chondrocytes in knee cartilage are stained brown. Based on this, there was no MGP expression in reference group chondrocytes. The chondrocytes in the 3-month group showed slight MGP positivity over a small area of cartilage surface. A deeper layer and larger area of MGP (+) chondrocytes was observed in the 6-month group, and in the 9-month to 12-month groups, MGP (+) chondrocytes were detected over the entire area of the cartilage selection, and went progressively deeper (Figs. 1 and 2). The IHC sections were independently analyzed by two independent investigators, and the two sets of results were in accordance and deemed credible $(\mathrm{ICC}=0.809, P<0.001)$.

To compare the mean IOD values (IHC) and the iTRAQ results, the Taylor series expansion method was used to calculate the expected values of the ratio of the data measured at each timepoint to the data measured at the first timepoint, and then the IOD data were proportionally converted according to the following derivation formula: 


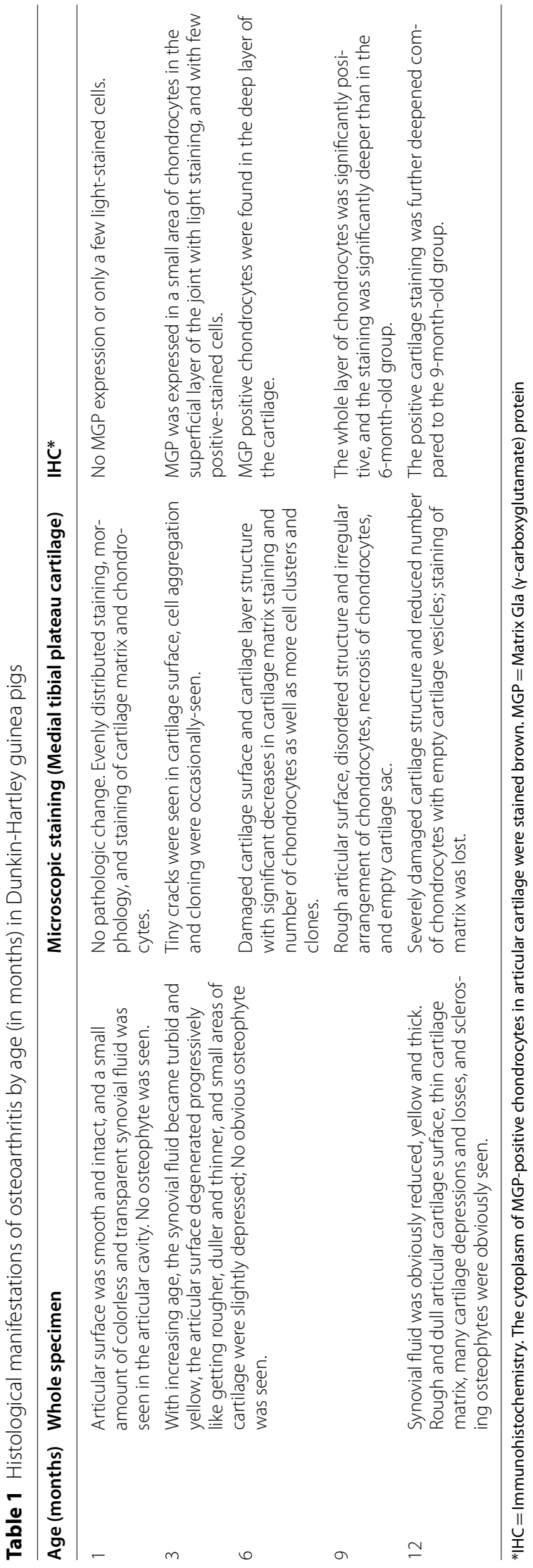


A

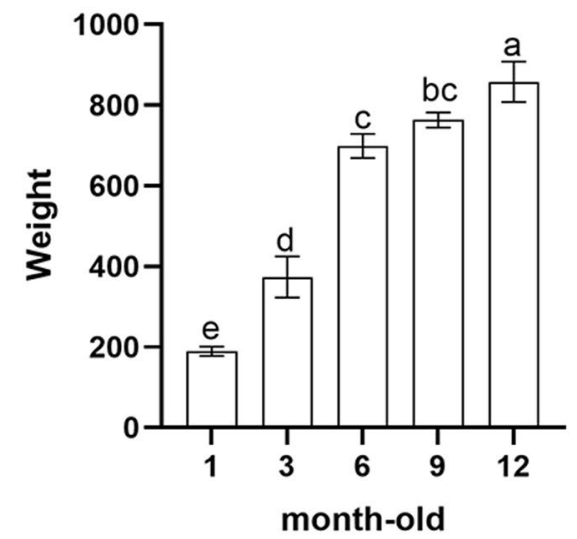

B

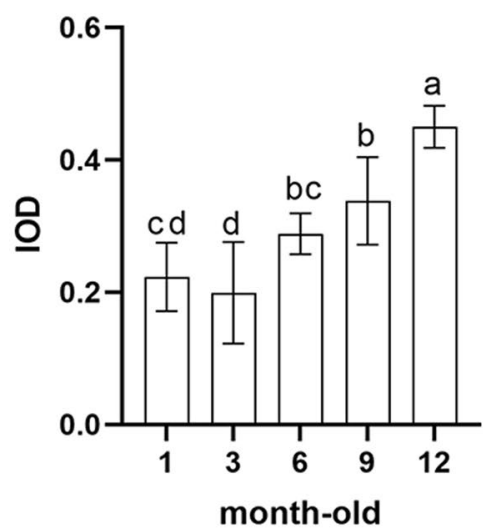

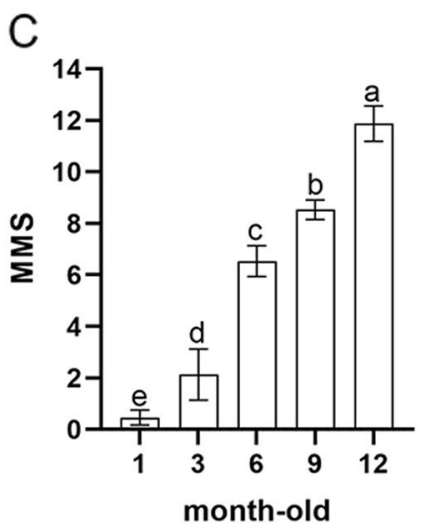

$\mathrm{D}$

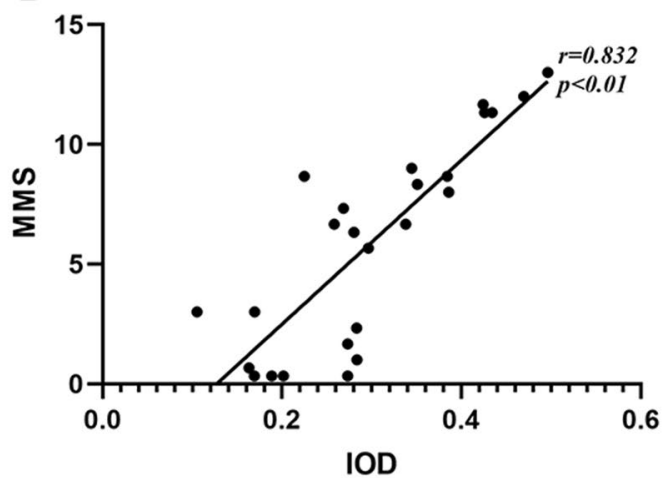

Fig. 2 Comparison of changes across month-age groups in the DH guinea pig spontaneous osteoarthritis model. A Weight changes with aging. The weight of the $\mathrm{DH}$ guinea pigs shows a generally escalating trend with increasing month-age. The month-to-month change is statistically significant where noted. B Modified Mankin Scores (MMS) of knee cartilages in every group. MMS tests show significant month-by-month increases with age in the DH guinea pigs. $\mathbf{C}$ In IHC analysis, MGP IOD values in every month-age. There is also a general trend of monthly increase of MGP expression in the joint in the aging DH guinea pigs. D The correlation analysis of MMS and MGP IOD of IHC analysis. The analysis confirms a significant positive correlation between MGP expression and OA severity (as MMS). * The same letter between different groups represented no significant difference

Table 2 Weights, outcomes of MMS and iTRAQ analysis of every month-age group

\begin{tabular}{|c|c|c|c|c|c|c|c|c|}
\hline Age (months) & Weight (g, Mean \pm SD) & MMS (Mean \pm SD) & $\mathrm{FC} 1 *$ & P1* & FC2 & P2 & FC3 & P3 \\
\hline 1 & $189.50 \pm 11.84$ & Reference & & & & & & \\
\hline 3 & $373.90 \pm 50.80$ & $2.13 \pm 0.99$ & 1.283 & 0.4750 & 1.039 & 0.9070 & 1.021 & 0.9087 \\
\hline 6 & $698.80 \pm 29.96$ & $6.53 \pm 0.60$ & 1.385 & 0.2831 & 1.287 & 0.0668 & 1.183 & 0.0592 \\
\hline 9 & $763.60 \pm 18.71$ & $8.53 \pm 0.38$ & 2.277 & 0.0437 & 2.033 & 0.0022 & 1.678 & 0.0045 \\
\hline 12 & $857.90 \pm 50.05$ & $11.87 \pm 0.69$ & 1.806 & 0.0259 & 1.205 & 0.0449 & 1.042 & 0.4457 \\
\hline
\end{tabular}

*FCs and Ps represented fold changes and $P$-values of every round of three-time iTRAQ 
Table 3 Original and transformed data of IOD

\begin{tabular}{|c|c|c|c|c|c|}
\hline \multirow[t]{2}{*}{ Method } & \multicolumn{5}{|c|}{ Month } \\
\hline & 1 & 3 & 6 & 9 & 12 \\
\hline \multicolumn{6}{|c|}{ Original data } \\
\hline IOD-Aver & 0.223 & 0.199 & 0.288 & 0.338 & 0.45 \\
\hline iTRAQ-G1* & 1 & 1.283 & 1.385 & 2.277 & 1.806 \\
\hline iTRAQ-G2 & 1 & 1.039 & 1.287 & 2.033 & 1.205 \\
\hline iTRAQ-G3 & 1 & 1.021 & 1.183 & 1.678 & 1.042 \\
\hline \multicolumn{6}{|c|}{ Transformed data } \\
\hline IOD-Trans & 1 & 0.916 & 1.443 & 1.520 & 2.182 \\
\hline iTRAQ-G1 & 1 & 1.283 & 1.385 & 2.277 & 1.806 \\
\hline iTRAQ-G2 & 1 & 1.039 & 1.287 & 2.033 & 1.205 \\
\hline iTRAQ-G3 & 1 & 1.021 & 1.183 & 1.678 & 1.042 \\
\hline
\end{tabular}

*iTRAQ $\mathrm{G} 1$ to $\mathrm{G} 3$ presented round 1 to round 3

$$
\left(\frac{x_{n}}{x_{1}}\right)=\frac{m_{n}}{m_{1}}+\frac{m_{n}}{m_{1}^{3}} D\left(x_{1}\right)-\frac{1}{m_{1}^{2}} \operatorname{Cov}\left(x_{1}, x_{n}\right)
$$

$\mathrm{m}_{1}$

$\mathrm{X}_{1}$

$\mathrm{D}\left(\mathrm{x}_{\mathrm{n}}\right)$

$\operatorname{CoV}\left(\mathrm{x}_{1}, \mathrm{x}_{\mathrm{n}}\right)$ is the mean of the first measurement time point and $M_{n}$ is that of the nth timepoint; is the observed value at the first measurement time point, $x_{n}$ is that of the $n$th time point;

is the variance of the nth measurement point;

The converted data from each group were used to check for correlation between the proportional change of the mean IOD values and the change trend of the iTRAQ data (Table 3). The correlation analysis confirmed a strong positive association (ICC >0.6) between the IOD measurements and the iTRAQ data trends (Fig. 3 and Supplementary Table 1). However, due to the small sample size, this finding did not reach significance.

\section{Discussion}

Besides potential strongly inhibiting ectopic calcifications in cartilage and cardiovascular tissues reported [22], MGP is reported to alleviate inflammation in OA [23]. MGP has been identified as a biomarker of OA [22], and a correlation between MGP and severity of degeneration of the nucleus pulposus has also been reported [24]. However, data regarding the link between MGP expression in articular cartilage and OA incidence and severity have been regarded as inconclusive. The results of the present study show that increasing MGP expression is significantly associated with aging and spontaneous progression of OA in this animal model; this is consistent with serum MGP findings in humans with OA [22].

MGP is a vitamin $\mathrm{K}$-dependent $\gamma$-carboxylated protein that has four known forms: completely carboxylated (cMGP), uncarboxylated (ucMGP), phosphorylated (pMGP), and non-phosphorylated (dpMGP) [25], all these forms can be detected by ELISA, but only total MGP, rather than any specific form, could be detected by IHC. MGP was synthesized in bone and by many mesenchymal cells, and is highly expressed in vascular smooth muscle cells and chondrocytes [26]. Although the detailed mechanisms of action of MGP remain unclear, it is thought to be related to the inhibition of BMP-2 and BMP-4 by blocking calcium crystal deposition [27]. Abnormal physiological regulation of MGP has also been reported in association with vascular calcification and urinary calculi, and it was recently reported that MGP is thought to be associated with angiogenesis [28] and chemoresistance [29] in tumors. It was reported that MGP may alleviate inflammation in acute pancreatitis and arthritis in 2011, but it was rarely studied in recent years.

DH guinea pigs are characterized by development of a spontaneous knee OA that can be used to simulate the pathogenesis OA in the human knee and thus could be considered a useful animal study model in spontaneous OA [30]. In this study, we first confirmed the onset of spontaneous $\mathrm{OA}$ in the $\mathrm{DH}$ guinea pigs by histopathological examination, and we found that the degeneration of the knee cartilage after 6-months of age was significant in terms of higher MMS compared with younger subgroups and reference group. This finding is consistent with previous studies. For primary validation of the changes in MGP expression, we performed iTRAQ and confirmed that the MGP fraction was significantly greater in older (9- and 12-month-old) subgroups than in younger and reference groups. IHC provided further validation of these results by showing increasing MGP expression in cartilage with age, and MGP levels of > 6-month-old subgroups were significantly higher than those of younger and reference groups, and generally increased with month-ages. Therefore, the overall results of the various parts of our study are consistent.

Although some previous studies have reported that higher MGP levels are linked to elevated incidence and severity of OA, the results remained inconclusive [31]. It has been reported that high serum MGP levels were associated with the incidence of osteoarticular diseases and that the severity of nucleus pulposus degeneration was also reflected by MGP, but these conclusions were not confirmed in subsequent studies [31-33], and even an opposite conclusion was reported in a GWAS study [34] that found lower MGP expression linked to a higher 


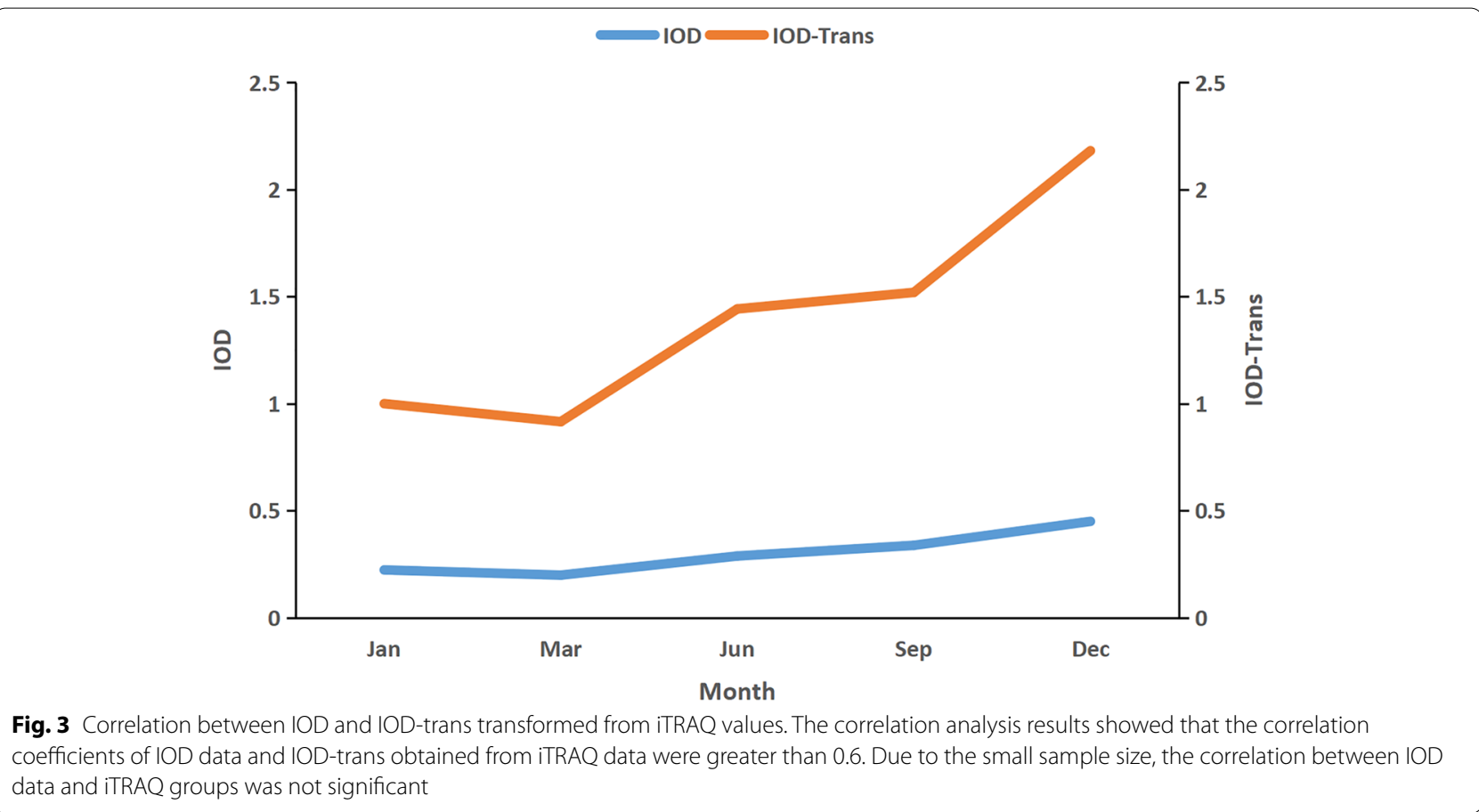

incidence of hand OA. In this study, a useful animal model of spontaneous and progressive OA was utilized and verified, and the results again showed that increased expression of MGP in male DH guinea pigs was present throughout aging and disease progression and may be link to increased OA severity. Severe inflammation and osteophyte formation are manifestations of advanced OA. Although MGP has a protective effect, its protective effect is limited and does not strong enough to reverse inflammation and ossification. It also indicates that more evidence is needed to confirm these outcomes and fully understand the association between MGP and OA. Further studies should be planned.

Together with previous studies, the outcomes of this study may provide valid new evidence toward understanding the association between changes in MGP level and severity of OA. To the best of our knowledge, this is the first study investigating MGP levels and every stage of spontaneous $\mathrm{OA}$ in $\mathrm{DH}$ guinea pig model. A major methodological strength of this study was that a longterm and continuous observation on the animal model was conducted, especially for the early stage of spontaneous OA, which is seldom seen in the previous studies. Secondly, MGP levels were assessed as DEPs by iTRAQ and validated by IHC at every fixed interval. A correlation analysis between the measured MGP levels and OA severity at histopathologic MMS was also performed, and a significant positive link was detected, further indicating that MGP may be a potential marker of OA progression and worthy for further investigation.

There were also several important limitations in this study. First, limited by study conditions, guinea pig strains without spontaneous OA, such as strain-13 and BS-2 guinea pigs [12, 35], which would be considered ideal reference group against DH guinea pigs, could not to be obtained. We had to use the 1-month-old guinea pigs of the same DH strain as the reference group to minimize possible bias as much as possible. And, we had to use the background staining of 1-month-old guinea pigs as negative reference group. Secondly, because the details of the association between MGP and OA have not been clarified, and the role of MGP in OA progression or alleviation may be complex, the upstream and downstream genes to MGP should be directly identified or manipulated. Therefore, gene-gene interaction studies are also necessary for further study of MGP and OA. In addition, only IOC was used for the IHC analysis, limited by the amount of extracted protein, and Western Blot (WB) and Real-Time Quantitative Reverse Transcription PCR (qRT-PCR) were not performed for verification.

In this study based on spontaneous $\mathrm{OA}$ in the $\mathrm{DH}$ guinea pig model, we have described associations between MGP expression and the natural history of degenerative joint disease. As $\mathrm{DH}$ guinea pigs have similar spontaneous OA natural history with human, especially in histopathological manifestations of osteoarthritic knees [16], MGP may be worthy for future studied. 
Our results suggest that higher MGP levels may be present in association with progression of OA. Additional rigorously designed studies will be needed to further investigate the role of MGP in OA.

\section{Conclusion}

Based on this study performed on male Dunkin-Hartley guinea pig spontaneous osteoarthritis model, our findings confirmed that elevated expression of MGP in DH guinea pigs may be concluded link to increased OA severity. Further studies are needed to investigate and confirm the association between MGP levels and OA severity.

\section{Supplementary Information}

The online version contains supplementary material available at https://doi. org/10.1186/s12891-021-04735-2.

\section{Additional file 1.}

Additional file 2.

Additional file 3.

\section{Acknowledgments}

We want to acknowledge Shengjing Hospital, the doctors and students, and all of the participants who contributed to this work, especially the staff of Animal Department of Shengjing Hospital Affiliated to China Medical University.

\section{Contribution of the authors}

Xueyong Liu designed the study. Xun Ma, Xinyuan Kang and Chunbo Deng performed the research, Yingwei Sun, Yanjun Li and Desheng Huang analyzed data, Chunbo Deng, Zhan Zhang and Xun Ma collected the data and checked the results. Zhan Zhang wrote and revised the paper. All authors read and approved the final manuscript.

\section{Funding}

This work was supported by Joint Program of Key Research and Development of Liaoning Province of China (2020JH2/10300138) and Natural Science Foundation of Liaoning Province (20180530044).

\section{Availability of data and materials}

All of the data in this study are obtained from experiments. The data used and analysed in this study are available from the corresponding author on reasonable request.

\section{Declarations}

\section{Ethics approval and consent to participate}

All animal experiments were performed with approval of the Animal Ethics Committee of Shengjing Hospital of China Medical University (2016PS284K), which complies with the Guide for the Care and Use of Laboratory Animals, 8th edition, published by the United States National Institutes of Health (NIH Publication, 2011); All methods in the present study were designed and performed in accordance with ARRIVE guidelines and the other relevant guidelines and regulations.

\section{Consent for publication}

This manuscript is approved by all authors for publication.

\section{Competing interests}

The authors declare no competing interests.

\section{Author details}

${ }^{1}$ Department of Rehabilitation, Shengjing Hospital of China Medical University, No.16, Puhe Street, Shenyang North New Area, Shenyang 110134, Liaoning Province, China. ${ }^{2}$ Department of Orthopaedics, Shengjing Hospital of China Medical University, Shenyang, Liaoning Province, China. ${ }^{3}$ Department of Orthopedics, Central Hospital of Shenyang Medical College, Shenyang, Liaoning Province, China. ${ }^{4}$ Department of Radiology, Affiliated Hospital of Liaoning University of Traditional Chinese Medicine, Shenyang, Liaoning Province, China. ${ }^{5}$ Department of Radiology, Shengjing Hospital of China Medical University, Shenyang, Liaoning Province, China. ${ }^{6}$ Department of Mathematics, College of Basic Medical Sciences, China Medical University, Shenyang, China.

Received: 19 April 2021 Accepted: 24 September 2021

Published online: 12 October 2021

\section{References}

1. Hunter D, Bierma-Zeinstra S. Osteoarthritis. Lancet (London, England). 2019;393(10182):1745-59. https://doi.org/10.1016/S0140-6736(19) 30417-9.

2. Safiri S, Kolahi A, Smith E, Hill C, Bettampadi D, Mansournia M, et al. Global, regional and national burden of osteoarthritis 1990-2017: a systematic analysis of the global burden of disease study 2017. Ann Rheum Dis. 2020;79(6):819-28. https://doi.org/10.1136/annrh eumdis-2019-216515.

3. Cancela L, Hsieh C, Francke U, Price P. Molecular structure, chromosome assignment, and promoter organization of the human matrix Gla protein gene. J Biol Chem. 1990;265(25):15040-8. https://doi.org/10.1016/S00219258(18)77221-9.

4. Shioi A, Morioka T, Shoji T, Emoto M. The inhibitory roles of Vitamin K in progression of vascular calcification. Nutrients. 2020;12(2). https://doi. org/10.3390/nu12020583.

5. Yao Y, Bennett B, Wang X, Rosenfeld M, Giachelli C, Lusis A, et al. Inhibition of bone morphogenetic proteins protects against atherosclerosis and vascular calcification. Circ Res. 2010;107(4):485-94. https://doi.org/10. 1161/CIRCRESAHA.110.219071.

6. Keutel J, Jörgensen G, Gabriel P. A new autosomal-recessive hereditary syndrome. Multiple peripheral pulmonary stenosis, brachytelephalangia, inner-ear deafness, ossification or calcification of cartilages. Deutsche medizinische Wochenschrift (1946). 1971;96(43):1676-81 passim. https:// doi.org/10.1055/s-0028-1110200.

7. Kusnierz CB, Gurda DA, Solnica B, et al. Serum matrix Gla protein concentrations in patients with mild and severe acute pancreatitis. Clin Lab. 2011:57:999-1006.

8. Silaghi CN, Fodor D, Cristea V, Craciun AM. Synovial and serum levels of uncarboxylated matrix Gla-protein (UCMGP) in patients with arthritis. Clin Chem Lab Med. 2011;50(1):125-8. https://doi.org/10.1515/CCLM.2011. 713.

9. Hui W, Cao Z, Wang X, Zhu J. Association of matrix Gla protein polymorphism and knee osteoarthritis in a chinese population. Biosci Rep. 2019:39(1). https://doi.org/10.1042/BSR20182228.

10. Shepherd C, Abigail E, Reynard Louise N, et al. Expression analysis of the osteoarthritis genetic susceptibility mapping to the matrix Gla protein gene MGP. Arthritis Res Ther. 2019;21:149. https://doi.org/10.1186/ s13075-019-1934-7.

11. Wang XJ, Wei L, Xue Y, et al. Experimental observation of the sequence of tibial plateau chondrocyte and matrix degeneration in spontaneous osteoarthritis in Guinea pigs. BMC Musculoskelet Disord. 2021;22:395. https://doi.org/10.1186/s12891-021-04281-x.

12. Huebner JL, Hanes MA, Beekman B, et al. A comparative analysis of bone and cartilage metabolism in two strains of guinea-pig with varying degrees of naturally occurring osteoarthritis. Osteoarthr Cartil. 2002;10:758-67. https://doi.org/10.1053/joca.2002.0821.

13. Bing W, Feng L. Attenuate Synovial Fluid Uncarboxylated Matrix Gla-Protein (ucMGP) Concentrations Are Linked with Radiographic Progression in Knee Osteoarthritis. Adv Clin Exp Med. 2015;24:1013-7. https://doi.org/ 10.17219/acem/33824.

14. Wallin R, Schurgers $L J$, Loeser RF, et al. Biosynthesis of the vitamin K-dependent matrix Gla protein (MGP) in chondrocytes: a fetuin-MGP 
protein complex is assembled in vesicles shed from normal but not from osteoarthritic chondrocytes. Osteoarthr Cartil. 2010;18:1096-103.

15. Worlein JM, Baker K, Bloomsmith M, Coleman K, Koban TL. The eighth edition of the GUIDE for the CARE and use of Laboratory animals (2011) implications for behavioral management. Am J Primatol. 2011;73:98-8.

16. Percie du Sert Nathalie,Hurst Viki,Ahluwalia Amrita et al: The ARRIVE guidelines 2.0: updated guidelines for reporting animal research. BMJ Open Sci, 2020, 4: e100115. https://doi.org/10.1136/bmjos-2020-100115.

17. Thomas NP, Wu WJ, Fleming BC, et al. Synovial inflammation plays a greater role in post-traumatic osteoarthritis compared to idiopathic osteoarthritis in the Hartley guinea pig knee. BMC Musculoskelet Disord. 2017;18:556. https://doi.org/10.1186/s12891-017-1913-6.

18. van der Sluijs JA, Geesink RG, van der Linden AJ, et al. The reliability of the Mankin score for osteoarthritis. J Orthop Res. 1992;10:58-61. https://doi. org/10.1002/jor.1100100107.

19. McGraw KO, Wong SP. Forming inferences about some intraclass correlation coefficients. Psychol Methods. 1996;1 (1):30-46. https://doi.org/10. 1037/1082-989X.1.1.30.

20. Kraus VB, Huebner JL, DeGroot J, Bendele A. The OARSI histopathology initiative - recommendations for histological assessments of osteoarthritis in the guinea pig. Osteoarthr Cartil. 2010;18(Suppl 3):S35-52. https://doi. org/10.1016/j.joca.2010.04.015.

21. Chen CH, Kuo SM, Tien YC, et al. Steady augmentation of anti-osteoarthritic actions of rapamycin by liposome-encapsulation in collaboration with low-intensity pulsed ultrasound. Int J Nanomedicine. 2020;15:377190. https://doi.org/10.2147/IJN.S252223.

22. Silaghi C, Fodor D, Gheorghe S, Crăciun A: Serum total matrix Gla protein: Reference interval in healthy adults and variations in patients with vascular and osteoarticular diseases. Clinica Chimica Acta; International Journal of Clinical Chemistry 2019, 490:128-134. 10.1016/j.cca.2018.12.029

23. Viegas C, Costa R, Santos L, Videira P, Silva Z, Araújo N, et al. Gla-rich protein function as an anti-inflammatory agent in monocytes/macrophages: Implications for calcification-related chronic inflammatory diseases. PLoS One. 2017;12(5):e0177829. https://doi.org/10.1371/journal.pone.0177829.

24. Chen S, Hu Z, Zhou Z, Lin X, Zhao F, Ma J, et al. Evaluation of 12 novel molecular markers for degenerated nucleus pulposus in a Chinese population. Spine. 2015;40(16):1252-60. https://doi.org/10.1097/BRS.00000 00000000929

25. Dalmeijer G, van der Schouw Y, Vermeer C, Magdeleyns E, Schurgers $\mathrm{L}$, Beulens J. Circulating matrix Gla protein is associated with coronary artery calcification and vitamin $\mathrm{K}$ status in healthy women. J Nutr Biochem. 2013:24(4):624-8. https://doi.org/10.1016/j.jnutbio.2012.02.012

26. Bjørklund G, Svanberg E, Dadar M, Card D, Chirumbolo S, Harrington D, et al. The role of matrix Gla protein (MGP) in vascular calcification. Curr
Med Chem. 2020;27(10):1647-60. https://doi.org/10.2174/0929867325 666180716104159

27. Yao Y, Zebboudj A, Shao E, Perez M, Boström K. Regulation of bone morphogenetic protein- 4 by matrix GLA protein in vascular endothelial cells involves activin-like kinase receptor 1. J Biol Chem. 2006;281 (45):3392130. https://doi.org/10.1074/jbc.M604239200.

28. Caiado H, Conceição N, Tiago D, Marreiros A, Vicente S, Enriquez J, et al. Evaluation of MGP gene expression in colorectal cancer. Gene. 2020:723:144120. https://doi.org/10.1016/j.gene.2019.144120.

29. Wu Y, Xia L, Guo Q, Zhu J, Deng Y, Wu X. Identification of chemoresistance-associated key genes and pathways in high-grade serous ovarian cancer by bioinformatics analyses. Cancer Manag Res. 2020;12:5213-23. https://doi.org/10.2147/CMAR.S251622.

30. Bendele A, Hulman J. Spontaneous cartilage degeneration in guinea pigs. Arthritis Rheum. 1988;31(4):561-5. https://doi.org/10.1002/art.17803 10416.

31. Misra D, Booth S, Crosier M, Ordovas J, Felson D, Neogi T. Matrix Gla protein polymorphism, but not concentrations, is associated with radiographic hand osteoarthritis. J Rheumatol. 2011;38(9):1960-5. https://doi. org/10.3899/jrheum.100985.

32. Shea M, Kritchevsky S, Hsu F, Nevitt M, Booth S, Kwoh C, et al. The association between vitamin $\mathrm{K}$ status and knee osteoarthritis features in older adults: the health, aging and body composition study. Osteoarthr Cartil. 2015;23(3):370-8. https://doi.org/10.1016/j.joca.2014.12.008.

33. Rutges J, Creemers L, Dhert W, Milz S, Sakai D, Mochida J, et al. Variations in gene and protein expression in human nucleus pulposus in comparison with annulus fibrosus and cartilage cells: potential associations with aging and degeneration. Osteoarthr Cartil. 2010;18(3):416-23. https://doi. org/10.1016/j.joca.2009.09.009.

34. den Hollander W, Boer C, Hart D, Yau M, Ramos Y, Metrustry S, et al. Genome-wide association and functional studies identify a role for matrix Gla protein in osteoarthritis of the hand. Ann Rheum Dis 2017;76(12):2046-53. https://doi.org/10.1136/annrheumdis-2017-211214.

35. Quasnichka H, Anderson-Mackenzie J, Bailey A. Subchondral bone and ligament changes precede cartilage degradation in guinea pig osteoarthritis. Biorheology. 2006;43:389-97. https://doi.org/10.1016/j.bbmt.2005. 09.011 .

\section{Publisher's Note}

Springer Nature remains neutral with regard to jurisdictional claims in published maps and institutional affiliations.

Ready to submit your research? Choose BMC and benefit from:

- fast, convenient online submission

- thorough peer review by experienced researchers in your field

- rapid publication on acceptance

- support for research data, including large and complex data types

- gold Open Access which fosters wider collaboration and increased citations

- maximum visibility for your research: over 100M website views per year

At BMC, research is always in progress.

Learn more biomedcentral.com/submissions 